\title{
Quality of life in pulmonary arterial hypertension
}

\author{
M.C. Post · J.J. Mager
}

Published online: 4 March 2015

(C) The Author(s) 2015. This article is published with open access at Springerlink.com

In the current issue of The Netherlands Heart Journal, Blok et al. describes the quality of life (QoL) in patients with pulmonary arterial hypertension (PAH) due to congenital heart disease (CHD) [1].

In this observational study, 39 out of 85 patients (46\%) with PAH-CHD who received PAH-targeted therapy filled in the Short-Form Health Survey (SF-36) QoL questionnaire correctly and could be included. Out of these 39 patients, $50 \%$ had Down syndrome. The diagnosis of PAH was based on echocardiography and defined as a tricuspid regurgitation velocity of at least $2.9 \mathrm{~m} / \mathrm{s}$ (estimated systolic pulmonary artery pressure (PAP) of $36 \mathrm{mmHg}$ ) [1].

PAH is a subgroup of pulmonary hypertension, including different forms with the same histopathological changes primarily affecting the pulmonary arteries, leading to endothelial dysfunction, proliferative changes and vascular remodelling [2]. The prevalence of PAH is between 15 and 50 cases per million [3]. The diagnosis should be confirmed by right heart catheterisation, and is characterised by a mean PAP of at least $25 \mathrm{mmHg}$ at rest and a pre-capillary haemodynamic profile including a pulmonary artery wedge pressure of less than $15 \mathrm{mmHg}$ and a pulmonary vascular resistance of more than 3 Woods Units [2]. Echocardiography is used to estimate the systolic PAP based on the tricuspid regurgitation peak velocity and the presence of additional echocardiographic variables suggestive of pulmonary hypertension. The presence of pulmonary hyperten-

M.C. Post $(\bowtie) \cdot$ J.J. Mager

Department of Cardiology and Pulmonology,

Center for Pulmonary Vascular Disease, St. Antonius Hospital,

3435 Nieuwegein/Utrecht, The Netherlands

e-mail: m.post@antoniusziekenhuis.nl sion is likely if the velocity is more than $3.4 \mathrm{~m} / \mathrm{s}$ (estimated systolic PAP $>50 \mathrm{mmHg}$ ) with or without additional variables [2]. Both underestimation and overestimation of the estimated systolic PAP frequently occur.

$\mathrm{PAH}$ is a debilitating and progressive disease with a tremendous psychosocial and economic impact for patients. A high prevalence of anxiety and depression (up to $40 \%$ ) is reported in patients with PAH leading to impairment of QoL [4]. An increase in clinical severity of the disease is associated with greater limitations and decrease in QoL. Exercise capacity, symptoms of right heart failure, haemodynamic characteristics, functional class and also mental disorders are associated with QoL. The majority of the studies on QoL in PAH patients used different standard generic questionnaires or tools specific for heart or lung diseases, not reflecting the clinical status of patients with $\mathrm{PAH}$ - for example the Minnesota Living with Heart Failure or the 36-item Medical Outcome Study Short-Form Health Survey Questionnaire (SF-36). Recently, a more disease-specific instrument was designed, the Cambridge PH Outcome Review (CAMPHOR) scale [5]. This scale exhibits superior psychometric properties, compared with the SF-36, in the assessment of pulmonary hypertension reported outcome [6].

In the study by Blok et al., almost all patients were on monotherapy receiving bosentan during follow-up [1]. In the 13 patients in whom the QoL declined during followup, the baseline functional class and exercise capacity were significantly worse, indicating more severe disease. No haemodynamic data (pulmonary vascular resistance or cardiac index) at baseline or changes in functional class during follow-up were reported. The 5-year survival rate was $82 \%$ and age, baseline functional class or exercise capacity and decline in QoL appeared to be predictors for mortality in this study. It is important to know that a decline in QoL is associated with worse outcome, i.e. higher mortality in 
PAH-CHD. However, it remains unclear whether the use of serial QoL questionnaires is superior or additive to the current follow-up strategy of evaluation of functional class, biomarkers and exercise capacity.

Besides general measures and supportive therapy, targeted PAH therapy is indicated in symptomatic patients with pulmonary vascular disease and a decreased functional capacity (Modified New York Heart Association functional class of at least II) $[2,7]$. The medical treatment of PAH is based on goal-oriented therapy [2]. The clinical response should be reassessed 3-6 months after the initial treatment and is based on different parameters or goals, such as: functional class, exercise capacity, biomarkers, echocardiography and haemodynamic parameters. If there is no improvement, sequential combination therapy should be started including another class of drugs [2]. Patients who achieve the treatment goals, irrespective of the type of medication used, seems to have a better prognosis and QoL. Predictors for outcome of PAH are sex, functional class, aetiology of PAH, exercise capacity, haemodynamics related to right ventricle performance and QoL [8].

CHD patients with an unrestricted pressure and volume overload due the presence of an intra- or extra-cardiac shunt are at risk for the development of PAH. However, PAH can develop at any stage of CHD, in the presence of a left-toright shunt but also up to years after a corrective procedure [9]. The extreme end of the PAH-CHD spectrum is the Eisenmenger syndrome, characterised by a reversed (right-to-left) or bidirectional shunt with severe cyanosis and multiorgan involvement. It is a rare disease and the number of patients will decrease in the next decades due to the improvement of CHD treatment in recent decades. Recent recommendations have stated that the defect should only be repaired if no pulmonary vascular disease is present, i.e. an indexed pulmonary vascular resistance of less than 4 Woods Units $\times \mathrm{m}^{2}[10$, 11]. The estimated prevalence of $\mathrm{PAH}$ in adults with $\mathrm{CHD}$ is $10 \%$, and it has an adverse impact on outcome and QoL [12]. Besides PAH-targeted therapy, individualised rehabilitation programmes are indicated for PAH-CHD patients to improve QoL by enabling participation in social activities and to maintain an active lifestyle [2,11]. Training leads to higher levels of physical activity, improved cardiorespiratory function and patient-reported QoL [13].

In patients with Down syndrome the prevalence of CHD, mainly atrioventricular septal defects, is about $50 \%$. These patients might be more prone to develop PAH compared with non-Down syndrome individuals with the same CHD. PAH-specific therapy seems to be safe and leads to increased exercise tolerance. However, the validity of measuring exercise capacity and QoL in this specific subgroup remains a concern [10].

Current guidelines on adult CHD and pulmonary hypertension recommend that patients with PAH-CHD should be followed by tertiary centres combining expertise in CHD and PAH in a multidisciplinary approach $[2,11]$.

\section{Conclusion}

PAH related to CHD is a severe disease with a significant impact on the QoL, as demonstrated by Blok et al. [1]. It appears that the serial measurements of QoL using validated questionnaires might be of additive value and a decline in QoL seems to be associated with an increased mortality in PAH-CHD.

Conflict of interest M.C. Post has received a speaker's fee from Actelion, Pfizer, and Bayer. J.J. Mager has no conflict of interest.

Open Access This article is distributed under the terms of the Creative Commons Attribution License which permits any use, distribution, and reproduction in any medium, provided the original author(s) and the source are credited.

\section{References}

1. Blok IM, Riel van AC, Schuuring MJ, et al. Decline in quality of life predics mortality in adult patients with pulmonary arterial hypertension due to congenital heart disease. Neth Heart J. 2015;23. doi:10.1007/s12471-015-0666-9

2. Galie N, Hoeper MM, Humbert M, et al. Guidelines for the diagnosis and treatment of pulmonary hypertension: the Task Force for the Diagnosis and Treatment of Pulmonary Hypertension of the European Society of Cardiology (ESC) and the European Respiratory Society (ERS), endorsed by the International Society of Heart and Lung Transplantation (ISHLT). Eur Heart J. 2009;30:2493-537.

3. Peacock AJ, Murphy NF, McMurray JJV, Caballero L, Stewart S. An epidemiological study of pulmonary arterial hypertension. Eur Respir J. 2007;30:104-9.

4. Guillevin L, Armstrong I, Aldrighetti R, et al. Understanding the impact of pulmonary arterial hypertension on patients' and carers' lives. Eur Respir Rev. 2013;22:535-42.

5. McKenna SP, Doughty N, Meads DM, Doward LC, Pepke-Zaba J. The Cambridge Pulmonary Hypertension Outcome Review (CAMPHOR): a measure of health-related quality of life and quality of life for patients with pulmonary hypertension. Qual Life Res. 2006; 15:103-15.

6. Twiss J, McKenna S, Ganderton L, et al. Psychometric performance of the CAMPHOR and SF-36 in pulmonary hypertension. BMC Pulm Med. 2013;13:45.

7. Schölzel BE, Snijder RJ, Mager JJ, et al. Chronic thromboembolic pulmonary hypertension. Neth Heart J. 2014;22:533-41.

8. Benza RL, Miller DP, Gomberg-Maitland M, et al. Predicting survival in pulmonary arterial hypertension: insights from the Registry to Evaluate Early and Long-Term Pulmonary Arterial Hypertension Disease Management (REVEAL). Circulation. 2010;122:164-72.

9. Dimopoulos K, Wort SJ, Gatzoulis MA. Pulmonary hypertension related to congenital heart disease: a call for action. Eur Heart J. 2014;35:691-700.

10. D'Alto M, Diller G-P. Pulmonary hypertension in adults with congenital heart disease and Eisenmenger syndrome: current advanced management strategies. Heart. 2014;100:1322-8. 
11. Baumgartner H, Bonhoeffer P, De Groot NMS, et al. ESC Guidelines for the management of grown-up congenital heart disease (new version 2010). Eur Heart J. 2010;31:2915-57.

12. Engelfriet PM, Duffels MGJ, Moller T, et al. Pulmonary arterial hypertension in adults born with a heart septal defect: the Euro Heart Survey on adult congenital heart disease. Heart. 2007;93:682-7.
13. Chan L, Chin LMK, Kennedy M, et al. Benefits of intensive treadmill exercise training on cardiorespiratory function and quality of life in patients with pulmonary hypertension. Chest. 2013;143:333-43. 